\title{
The simulation for study of von Mises stress sensitiveness to the change of coronal conformity in knee prosthesis
}

\author{
Usman $^{1}$, Shyh Chour Huang ${ }^{2}$ \\ ${ }^{1,2}$ National Kaohsiung University of Applied Sciences, Mechanical Engineering Department, 80778 Kaohsiung, Taiwan, R.O.C.
}

\begin{abstract}
Two-dimensional finite element contact modelling is often conducted to simulate the contact mechanism in knee prosthesis. This is based on the assumption that the contact stresses are more sensitive to change in the coronal plane than in the sagittal plane. Therefore, the simulation is then can be simply conducted only in the coronal plane with neglecting the sagittal dimension. Thus, the model is only a two-dimensional model. Based on this issue, the investigation of the response of contact stress (von Mises) to the coronal and sagittal conformity variation was conducted. For the need of investigation, ten three-dimensional finite element models of knee prosthesis have been created. From the result, the gradient of von Mises stress when the conformity varied in the coronal plane was -12.015 , and when the conformity was varied in the sagittal plane, the gradient was -9.774 . This meant that the von Mises stress had high sensitiveness to the change of the coronal conformity variation of knee prosthesis than to the change of the sagittal conformity. Therefore, the contact modelling of knee prosthesis can be simply carried out in the coronal plane.
\end{abstract}

\section{Introduction}

Contact stresses in knee prosthesis are more sensitive to change in coronal plane [1] than in sagittal plane. Therefore, the investigation of contact stresses in knee prosthesis is said simply can be carried out only in the coronal plane, without involving the sagittal plane. Thus, the contact stresses in knee prosthesis can simply be attained by only two-dimensional simulation which only simulated in the coronal plane. Therefore, the purpose of this research is to examine the response of von Mises stress, as one of the contact stresses, to coronal and sagittal conformity variation. Ten finite element models of knee prosthesis had been created for the investigation.

\subsection{Knee joint}

Among the joints in the human body, the knee joint is the largest and the most complicated joint [2]. The contact category in a knee joint is non-conforming. The nonconforming articular counterface leads to a point contact, which is a knee joint load is applied to a small area. Therefore, the high contact pressure occurs in the knee joint.

There are four bones make up the knee joint. Two of them are the thigh bone and shin bone, which are so called femoral and tibial bone, respectively. The other two are the fibula and kneecap bone (patella). The fibula is positioned along the lateral side of the shin bone [2]. And the patella as its knee joint shielding function, it is located in front of the knee joint [3]. The knee joint bones aforementioned are connected each other by mean of the ligaments. And for the need of joint movement, they are also linked by the tendons to the leg muscles [2].

There are three ligaments in the knee joint; the anterior cruciate ligament, the posterior cruciate ligament, the medial collateral ligament, and the lateral collateral ligament. The anterior and posterior cruciate ligaments avoid the thigh bone to dislocate posteriorly and anteriorly, respectively. And the medial and lateral collateral ligaments prevent the thigh bone to slide laterally and medially, respectively [2]. In some knee prostheses design, both the anterior and posterior cruciate ligaments are removed. Instead, to constraint the knee prosthesis from sliding anteroposteriorly and mediolaterally, the stabilizer is created. Besides, the cam shape of knee prosthesis also behaves as anterior and posterior cruciate ligaments. Meanwhile, in some other knee prostheses design, the cruciate ligaments are retained to keep natural stability. For a cushion and shock absorber in the knee joint, the medial and lateral menisci exist. In total knee arthroplasty, the menisci are removed and the cushion and shock absorber task is substituted by the insert.

\subsection{Indication for total knee arthroplasty}

Severe damage of the knee joint due to the osteoarthritis, the rheumatoid arthritis or some other knee joint degenerative diseases, indicates the total knee arthroplasty. The main objective of the total knee arthroplasty is to bring back the knee joint loss function 
due to severe knee arthritis that inflicts a serious pain and loss of stability of the knee joint. Otherwise, they can reduce the quality of life, or indeed, to some extent, they can disable the patient. The total knee arthroplasty is usually performed to older patients, but the procedure may also be implemented to young people considering that young people also have the possibility of suffering from the degenerative knee joint diseases [4].

Three among the many knee joint degenerative diseases are the osteoarthritis, the rheumatoid arthritis, and the post-traumatic arthritis. The osteoarthritis is induced by continued wear and damage of knee joint. The rheumatoid arthritis is occurred due to the inflammation of the membrane encompassing the knee joint. Both osteoarthritis and rheumatoid arthritis, at a certain level, tear the knee join cartilage. And thenceforth, the patients undergo a serious pain. Post-traumatic arthritis is the arthritis generated by harsh knee joint trauma. The bone fracture and ligament damages can lacerate the cartilage [4].

From these explanations of knee joint diseases, the serious pains in the knee joint appear after cartilage deterioration. This is because they are cartilages (distal femoral and proximal tibial cartilage) that rub each other during human locomotion. Therefore, the cartilage damage is a reason for the total knee arthroplasty.

\subsection{Knee prosthesis}

There are two popular designs of the knee prosthesis; the posterior-stabilized and the cruciate-retaining prostheses design. In the posterior-stabilized prostheses design, both anterior and posterior cruciate ligament are removed. The cruciate ligament functions are, then, substituted by the stabilizer and cam mechanism created in the knee prosthesis. Meanwhile, the cruciate-retaining prostheses design maintains the cruciate ligament on the existing position [4].

There are three main parts in knee prosthesis: condyle, insert, and tray (Figure 1). Condyle and tray are made of metal, and insert is made of polyethylene. Condyle is mounted on distal femur and tray is on proximal tibia. Meanwhile, the insert serves as a cushion between condyle and tray. When the condyle is brought into contact with the insert, the contact area will occur around the contact region. There are two kinds of contact area in knee prosthesis contact mechanism: initial contact area before deflection when there is not load is applied to the knee prosthesis; and the contact area after deflection when load is applied. The initial contact area is a result of conformity. Meanwhile, the contact area after deflection is a result of insert deformation.

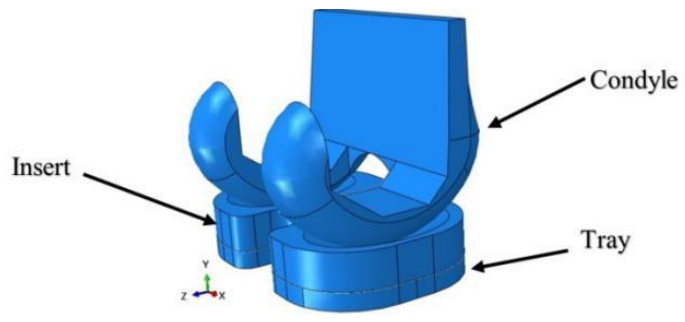

Figure 1. Parts of knee prosthesis

\section{Materials and methods}

The preprocession, simulation, and postprocession are three stages of finite element analysis in Abaqus software. The preprocession is the stage when the models are defined. There are seven steps have to be determined in this stage: creating the parts, defining the material property, parts assembly, deciding the step size, making the interaction between parts, applying loads, and discretizing the parts (meshing). In the simulation stage, the numerical problems of the model are solved. The time needed for the simulation depends on the complexity of models. The results of simulation are, then, evaluated in the postprocession stage [5].

Solidworks software was used to create the models and subsequent analysis was carried out in Abaqus finite element analysis software by importing the models into it. The coronal radius of condyle was $23 \mathrm{~mm}$ and the sagittal radius was $37 \mathrm{~mm}$ [6]. The condyle dimensions were not varied. Instead, to obtain the difference value of contact articular conformity, the radius of insert for both in coronal and sagittal plane was varied.

Due to the focus of our design was a symmetric knee prosthesis, that is the lateral and medial section of knee prosthesis are equal in size, we simplified the knee prosthesis model by only taking the medial section of knee prosthesis. We chose the medial section in the analysis due to the fact that the medial section supports $60 \%$ of total load applied to knee joint [7].

We used the knee joint absolute maximum load of four times bodyweight which occurred on the activity of descending stair [8]. And, the bodyweight of $700 \mathrm{~N}$ was chosen. Thus, the normal load yielded $1680 \mathrm{~N}$

When the conformity in sagittal plane was varied, the coronal conformity was set to be maximum value at 1 (full conforming). And conversely, when the conformity in the coronal plane was varied, the sagittal conformity was set to be maximal. The conformity was varied from 0.2 until 1 with interval of 0.2 , namely $0.2,0.4,0.6,0.8$, and 1 .

The condyle was assumed as a rigid body due to its much higher elastic modulus compared with UHMWPE as the material of the insert $(\mathrm{E}=550 \mathrm{MPa})$, and the Poisson's ratio of UMWPE of 0.44 was chosen [9]. The nonlinear behavior of true stress and the corresponding true strain of UHMWPE model are given in Figure 2. The friction coefficient of 0.04 was chosen [10].

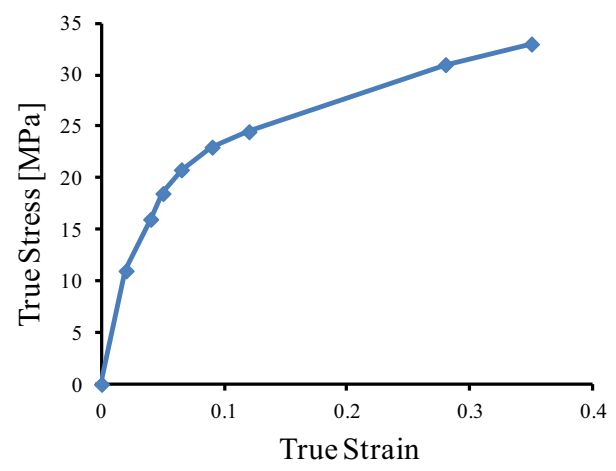

Figure 2. The nonlinear behavior of stress-strain of UHMWPE [10]. 
The condyle models, as a rigid body, were meshed with four-noded bilinear rigid quadrilateral element with global edge size of $0.75 \mathrm{~mm}$. Meanwhile, the inserts, as a deformable body, were mashed with eight-noded linear brick element with global edge size of $1 \mathrm{~mm}$. One of the finite element models for both condyle and insert models are shown in Figure 3.

\section{Results and discussions}

Figure 4 shows the conformity versus the von Mises stress for the coronal and sagittal conformity variation. The figure depicts the von Mises stress versus conformity equipped with the linear regression line. By the regression line, we know that the sensitiveness of von Mises stress to the variation of the coronal conformity was greater (with gradient of -12.015) than that occurred when conformity was varied in the sagittal plane, where the gradient $(-9.774)$ was smaller than that found when the coronal conformity was varied.

The results assured us that the contact simulation in knee prosthesis can be decisively done by using simple two-dimensional simulation in the coronal plane. This can reduce not only the complexity of creating the biomechanical model -which is very difficult to create with the existing software because the shape is always irregular, but also can reduce the computation time and cost.

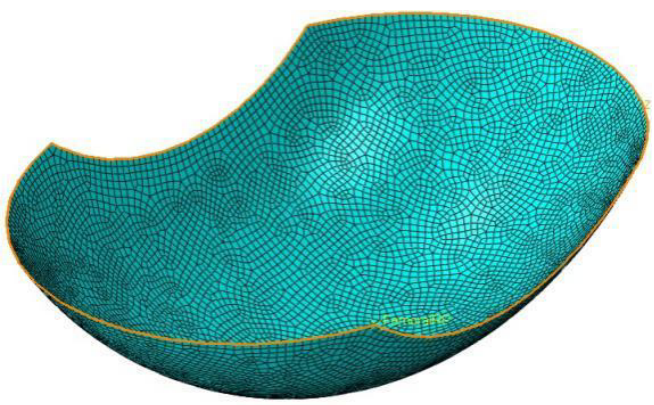

a)

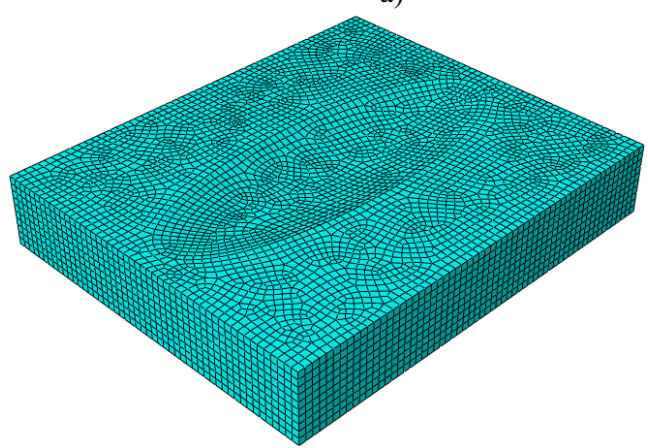

b)

Figure 3. a) Condyle model with bilinear rigid quadrilateral element; (b) Insert model with eight-noded linear brick element.

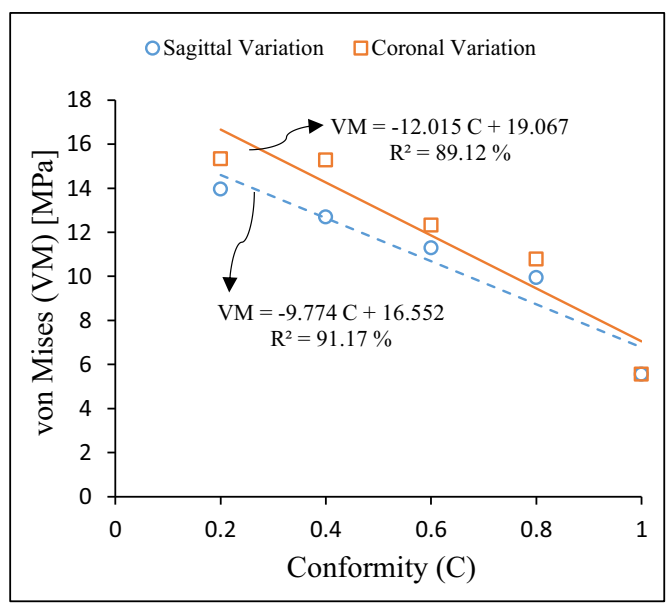

Figure 4. The von Mises stress versus the coronal and sagittal conformity variation with linear regression line.

Figure 5 shows the von Mises stress in insert model at lowest conformity level. The most important phenomenon that has to be noted here was the highest von Mises stress took place at the edge of insert dish. Meanwhile, at the center of contact, the highest von Mises stress took place below the insert surface. This meant that the sharp edge in the insert edge dish design has to be avoided. These phenomena existed in both coronal and sagittal conformity variation simulation.

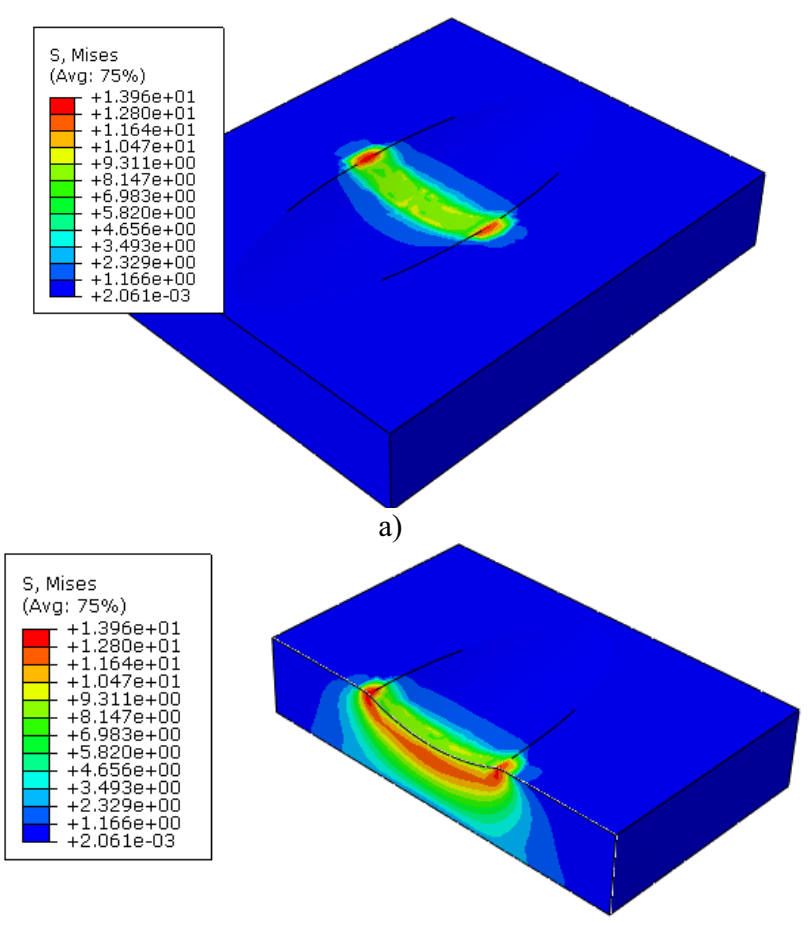

b) 


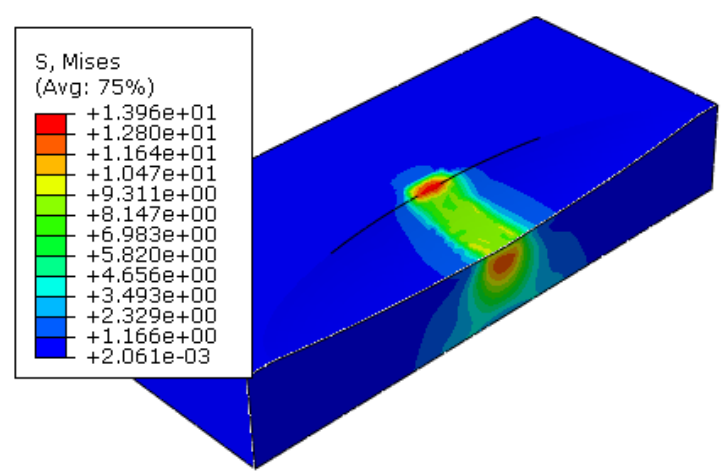

c)

Figure 5. a) One of the insert models with the von Mises stress appears at the surface; b) The coronal cut view of the insert model. It shows that the highest von Mises stress occurred below the surface along the coronal plane; c) The sagittal cut view of the insert model.

\section{Conclusions}

The investigation for proving the sensitiveness of the contact stress to change in the coronal plane has been conducted. We have revealed that the coronal variation of the conformity gave a more considerable increasing in von Mises stress, as one of contact stresses, than that given by the sagittal conformity variation.

Therefore, the study of the contact stresses in knee prosthesis can be only simulated in the coronal plane. So that, we can save time and computation cost because the simulation is conducted in only two-dimensional analysis.

\section{Acknowledgement}

The authors acknowledge and thank the Ministry of Science and Technology of the Republic of China for their partial financial support of this study with the Contract No. MOST 105-2221-E-151-016.

\section{References}

1. J. J. Rawlinson, D. L. Bartel, J Biomech 35, 27-34 (2002).

2. Information on http://www.webmd.com.

3. Information on http://orthoinfo.aaos.org.

4. S. Affatato, The history of total knee arthroplasty (TKA) (Elsevier Ltd., 2015).

5. Simulia, Getting Started with Abaqus: Interactive Edition (Dassault Systèmes, 2014).

6. Y. F. Huang, S. C. Huang, Optimal Shape Wear Analysis of Total Knee Replacement (Department of Mechanical Engineering, National Kaohsiung University of Applied Sciences, Master Thesis, 2015).

7. E. A. Morra, A. S. Greenwald, J Bone Joint Surg. 85, $111-114$ (2003).

8. I. Kutzner, B. Heinlein, F. Graichen, A. Bender, A. Rohlmann, A. Halder, A. Beier, G. Bergmann, J Biomech 43, 2164-2173 (2010).

9. R. Willing, I. Y. Kim, J Biomech 42, 2520-2527 (2009).

10. M. Bahraminasab, B.B. Sahari, M. R. Hassan, M. Arumugam, M. Shamsborhan, Trends Biomater Artif Organs 25, 95-100 (2011). 